NYU-TH/01/04/01

SNS-PH/01-04

hep-th/0104065

\title{
On the Absorption by Near-Extremal Black Branes
}

\author{
G. Policastro ${ }^{a, b}$ and A. Starinets ${ }^{a}$ \\ (a) Department of Physics, New York University, \\ 4 Washington Place, New York NY 10003 USA \\ (b) Scuola Normale Superiore, \\ Piazza dei Cavalieri 7, 56100, Pisa, Italy
}

\begin{abstract}
We study the absorption of a minimally coupled scalar in the gravitational background created by a stack of near-extremal black three-branes, and more generally by $M 2, M 5$ and $D p$ branes. The absorption probability has the form $P(l)=P_{0}(l) f_{l}(\lambda)$, where $P_{0}(l)$ is the partial wave's absorption probability in the extremal case, and the thermal factor $f_{l}(\lambda)$ depends on the ratio of the frequency of the incoming wave and the Hawking temperature, $\lambda=\omega / \pi T$. Using Langer-Olver's method, we obtain a low-temperature $(\lambda \gg 1)$ asymptotic expansion for $P(l)$ with coefficients determined recursively. This expansion, which turns out to be a fairly good approximation even for $\lambda \sim 1$, accounts for all power-like finite-temperature corrections to $P_{0}(l)$, and we calculate a few terms explicitly. We also show that at low temperature the absorption probability contains exponentially suppressed terms, and attempt to develop an approximation scheme to calculate those. The high-temperature expansion is also considered. For the $s$-wave, the low-temperature gravity result is consistent with the free finite-temperature field theory calculation, while for high temperature and higher partial waves we find a disagreement. As a check of the approximation methods used, we apply them to the $D 1-D 5$-brane system, and compare results to the known exact solution.
\end{abstract}




\section{Introduction}

It is believed that a strongly coupled gauge theory at finite temperature can be studied using its gravity dual description in the framework of the AdS/CFT correspondence. Examples relevant for $d=4$ gauge theories include Witten's interpretation of the confinementdeconfinement phase transition in terms of the Hawking - Page transition in AdS and calculation of the free energy of the finite-temperature $\mathcal{N}=4$ SYM which matches (up to a factor of $4 / 3$ ) the free energy of the near-extremal three-branes (for review and references see section 3.6 of [四]).

Calculation of the absorption cross section by $D 3$-branes revealed a remarkable agreement between conformal field theory and gravity results, obtained, correspondingly, at weak and strong 't Hooft coupling [2]. The agreement has been explained (at least for the $s$-wave absorption) by establishing a non-renormalization theorem enjoyed by certain $\mathcal{N}=4$ SYM correlation functions [3], and serves as one of the few direct checks of the conjectured AdS/CFT correspondence. Supersymmetry of the D3-branes' world-volume theory is crucial for the existence of the above mentioned non-renormalization theorem. At finite temperature the supersymmetry is broken, and one cannot expect the results computed at strong and weak coupling to agree.

However, for the $D 1 / D 5$ model of a five-dimensional black hole the agreement between greybody factors and the entropy calculated in two-dimensional CFT and in gravity holds even in the absence of supersymmetry. The absorption cross-section has the form $\sigma=\sigma_{0} f\left(\omega, T_{L}, T_{R}\right)$, where $\sigma_{0}$ is the cross-section at zero temperature and the thermal factor $f\left(\omega, T_{L}, T_{R}\right)$ can be computed either from (super)gravity (in the low energy regime, i.e. when $\left.\omega r_{1} \ll 1, \omega r_{5} \ll 1\right)$ or by considering a finite-temperature two-point function in the dual CFT [1], [5], [6]. A perfect matching between CFT and gravity results can be explained by arguing that at sufficiently low energies the excitations of the $D 1 / D 5$ system are correctly described by the moduli space approximation even at strong coupling [7].

A priori, the existence of a non-renormalization theorem not related to supersymmetry in $d=4$ can be considered as an extremely remote possibility, and indeed in this paper we do not find an exact agreement between gravity and weakly coupled field theory calculations. However, the details of the disagreement are intriguing enough, and in the spirit of AdS/CFT we regard our results obtained from gravity as a prediction for a strongly coupled finite-temperature gauge theory.

For the D1-D5-brane system, it is fairly straightforward to calculate the thermal factor $f\left(\omega, T_{L}, T_{R}\right)$ on the gravity side, since in the low energy regime the exact solution of the corresponding wave equation is known. This is not the case for the generic black brane solution, and for the three-brane solution corresponding to finite temperature $d=4$ gauge theory in particular. It is a purpose of this paper to develop a reliable approximation scheme which would allow one to calculate greybody factors for the case of a non-extremal 
three brane, and more generally for $M 2-, M 5$ - and $D p$-branes. Earlier attempts to calculate the absorption probability for non-extremal three-branes were made in [8], [9], [10]. However, we will show that only the zero-temperature limit of the results obtained there is correct.

In the low energy regime $(\omega R \ll 1)$ the radial part of the wave equation in a typical black-brane background has several regular singular points. Three of them always correspond, respectively, to the position of outer, inner horizons and spatial infinity, and the rest lies on a unit circle in the complex plane. Since the number of singular points exceeds three, the exact solution is unaccessible. However, the equation contains a parameter $\lambda=\omega / \pi T$ making it possible to study "low-temperature" $(\lambda \gg 1)$ and "high-temperature" $(\lambda \ll 1)$ asymptotics. While obtaining the high-temperature asymptotics is a straightforward application of perturbation theory, the low-temperature expansion requires a careful WKB analysis of the differential equation in the presence of singular points. Basic principles of such an analysis have been developed a long time ago in [11, 12] (see also [13] and a classic book by Olver [14]). Using Langer - Olver's method, we are able to obtain uniform asymptotic expansions (with coefficients determined recursively) for the solutions of the wave equation. The uniformity of approximation is vital for the correct calculation of the absorption probability. Olver's theorem guarantees this property only when the coefficients and the corresponding error-control function converge everywhere on the interval of consideration. For non-extremal branes this interval always contains two singular points (a situation more general than the one originally treated in [11, [12]), and therefore the convergence should be checked in each case independently. Once the convergence has been established, all power-like corrections to the zero-temperature (extremal) result can in principle be determined using the recursive relations. We obtain explicit asymptotic expansions for the black three-brane background (Section 3.1) and for non-extremal D1-D5-brane system (Section 国), and demonstrate (in Section 6) that all $M$ - and black-brane backgrounds can be treated using this approach. Being an asymptotic expansion in powers of $1 / \lambda$, the method cannot account for the contributions of the form $\exp (-\lambda)$ (and for the $s$-wave those are the only ones present). In Section 3.2 we make an attempt to build an approximation scheme based on the hypergeometric function which would allow the calculation of these exponentially small contributions. We find the leading term of the corresponding expansion, but the subleading corrections are controlled by an integral equation which we were unable to solve. Section 3.3 deals with the high-temperature expansion. In Section 5 we compute the absorption cross section in field theory using the method of finite-temperature Green's functions. Appendices A, B, Q contain some relevant technical details. 


\section{Summary of results}

Our main result can be formulated as follows: the absorption probability for a minimally coupled massless scalar in the gravitational background created by a stack of near-extremal black three-branes can be written as $P(l)=P_{0}(l) f_{l}(\lambda)$, where

$$
P_{0}(l)=\frac{4 \pi^{2}}{(l+1) !^{2}(l+2) !^{2}}\left(\frac{\omega R}{2}\right)^{4 l+8}
$$

is the absorption probability for the extremal D3-branes. The absorption cross section (greybody factor) for the $l$-th partial wave is related to the absorption probability $P_{l}$ via

$$
\sigma_{l}=\frac{2 \pi^{\frac{d-1}{2}} \Gamma(l+d-2)(l+d / 2-1)}{\omega^{d-1} \Gamma(d / 2-1 / 2) \Gamma(l+1)} P(l),
$$

where $d$ is the number of dimensions transverse to the brane 15. For the thermal factor $f_{l}(\lambda)$ we obtain a low-temperature $(\lambda=\omega / \pi T \gg 1)$ asymptotic expansion

$$
\begin{aligned}
f_{l}(\lambda) & =1+\frac{2 \nu\left(\nu^{2}-1\right)\left(\nu^{2}-4\right)}{5 \lambda^{4}} \\
& +\frac{2 \nu\left(\nu^{2}-1\right)\left(\nu^{2}-4\right)(\nu-3)\left(9 \nu^{4}-23 \nu^{3}-114 \nu^{2}+188 \nu+600\right)}{225 \lambda^{8}} \\
& +O\left(1 / \lambda^{12}\right),
\end{aligned}
$$

where $\nu=l+2$. The function $f_{l}(\lambda)$ for $\lambda \gg 1$ also contains exponentially small terms (see $(3.39))$. The high-temperature limit of $f_{l}(\lambda)$ for various $l$ is given by (3.47), (3.48), (3.49). In particular, for the $s$-wave the gravity calculation gives

$$
f_{0, \text { grav }}(\lambda)= \begin{cases}1+O\left(e^{-\lambda}\right) & \text { for } \lambda \gg 1, \\ \frac{8}{\pi \lambda^{3}}\left(1+\frac{\left(\pi^{2}+12(2-\log 2) \log 2\right) \lambda^{2}}{48}+\cdots\right) & \text { for } \lambda \ll 1,\end{cases}
$$

while in field theory we get $f_{0, \text { field th. }}(\lambda)=\operatorname{coth}(\pi \lambda / 4)$, i.e.

$$
f_{0, \text { field th. }}(\lambda)= \begin{cases}1+O\left(e^{-\lambda}\right) & \text { for } \lambda \gg 1, \\ \frac{4}{\pi \lambda}\left(1+\frac{\pi^{2} \lambda^{2}}{48}+\cdots\right) & \text { for } \lambda \ll 1 .\end{cases}
$$

For $l=1$ the disagreement is visible already at low temperature,

$$
\begin{aligned}
f_{0, \text { grav }}(\lambda) & =1+48 / \lambda^{4}+O\left(e^{-\lambda}\right), \\
f_{0, \text { field th. }}(\lambda) & =1+8 / \lambda^{2}+48 / \lambda^{4}+32 / \lambda^{6}+O\left(e^{-\lambda}\right) .
\end{aligned}
$$

As mentioned above, we do not expect free field theory and gravity results to agree, especially in the regime $\omega \ll T$, where the resummation of an infinite subset of diagrams in the finite-temperature gauge theory is required. We rather regard (2.4) as a prediction for a strongly coupled gauge theory at non-zero temperature. 


\section{Absorption by non-extremal black three-branes}

The metric of the black three-brane is given by [16]

$$
d s_{10}^{2}=\frac{-f(r) d t^{2}+d \vec{x} \cdot d \vec{x}}{\sqrt{H(r)}}+\sqrt{H(r)}\left(\frac{d r^{2}}{f(r)}+r^{2} d \Omega_{5}^{2}\right),
$$

where

$$
H(r)=1+\frac{R^{4}}{r^{4}}, \quad f(r)=1-\frac{r_{0}^{4}}{r^{4}} .
$$

The position of the inner horizon corresponds to $r=0$, the outer horizon is located at $r=r_{0}$. We are interested in the region of the parameter space characterized by $\omega R \ll 1, T R \ll 1$ with the ratio $\lambda=\omega / \pi T$ remaining arbitrary. The condition $T R \ll 1$ implies that the metric we consider is near-extremal $\left(r_{0} \ll R\right)$. For $r_{0} \ll R$ the Hawking temperature is related to the non-extremality parameter $r_{0}$ by $T=r_{0} / \pi R^{2}$. The horizon area is given by $A_{H}=\pi^{3} r_{0}^{5} \sqrt{H\left(r_{0}\right)} \rightarrow \pi^{3} r_{0}^{3} R^{2}$ for $r_{0} \ll R$.

Using $\rho=\omega r, \rho_{0}=\omega r_{0}$, one can write the radial part of the Laplace equation for a minimally coupled massless scalar as

$$
\frac{d^{2} \phi}{d \rho^{2}}+\frac{5 \rho^{4}-\rho_{0}^{4}}{\rho\left(\rho^{4}-\rho_{0}^{4}\right)} \frac{d \phi}{d \rho}-\frac{\rho^{2} l(l+4)}{\rho^{4}-\rho_{0}^{4}} \phi+\frac{\rho^{4}\left(\rho^{4}+(\omega R)^{4}\right)}{\left(\rho^{4}-\rho_{0}^{4}\right)^{2}} \phi=0 .
$$

In the extremal case $r_{0}=0$, equation (3.2) in terms of $z=r^{2}$ reduces to

$$
4 z^{3} \phi^{\prime \prime}+12 z^{2} \phi^{\prime}+\left(\omega^{2} R^{2}-l(l+4) z+\omega^{2} z^{2}\right) \phi=0,
$$

solutions of which are known'. Exact solutions of (3.2) are not known, and therefore one must seek a reliable approximation scheme. Introducing $x=r_{0}^{2} / r^{2}$, we can write (3.2) as

$$
\frac{d^{2} \phi}{d x^{2}}-\frac{\left(1+x^{2}\right)}{x\left(1-x^{2}\right)} \frac{d \phi}{d x}-\frac{l(l+4)}{4 x^{2}\left(1-x^{2}\right)} \phi+\frac{\left(\lambda^{2}+\rho_{0}^{2} / x^{2}\right)}{4 x\left(1-x^{2}\right)^{2}} \phi=0 .
$$

Following [2] we shall calculate the absorption probability to the leading order in $\omega R \ll 1$ by introducing the "inner" and "outer" regions for the equation (3.4), and matching the corresponding solutions. In the outer region, $\rho \gg \rho_{0}, \rho \gg(\omega R)^{2}$, equation (3.2) reduces to a Bessel equation with the solutions

$$
\phi(r)=\alpha J_{l+2}(\rho) / \rho^{2}+\beta Y_{l+2}(\rho) / \rho^{2} .
$$

In the inner region, $\rho_{0} \leq \rho \ll 1$, the last term in (3.4), $\rho_{0}^{2} / x^{3}$ can be neglected in comparison with the typical| "potential" term $\sim 1 / x^{2}$. Equation (3.4) becomes

$$
\frac{d^{2} \phi}{d x^{2}}-\frac{\left(1+x^{2}\right)}{x\left(1-x^{2}\right)} \frac{d \phi}{d x}-\frac{l(l+4)}{4 x^{2}\left(1-x^{2}\right)} \phi+\frac{\lambda^{2}}{4 x\left(1-x^{2}\right)^{2}} \phi=0 .
$$

\footnotetext{
${ }^{1}$ See 2.306 of [17]. A complete absorption analysis based on the exact solution has been done in [18].

${ }^{2}$ The term with $l$ is an example of such a typical term. However, even for $l=0$ the "potential" characterizing the geometry is proportional to $1 / x^{2}$ - this can be seen by converting the equation into the Schrödinger form.
} 
It has four regular singular points (at $x= \pm 1,0, \infty$ ). Unlike the case of a second order differential equation with three regular singular points which can always be cast into the form of hypergeometric equation by performing a suitable conformal transformation, such an equation has no common ancestor and should be studied on its own. The equation, however, contains a parameter $\lambda$ making it possible to study low- and hightemperature expansions even though the exact solution remains unaccessible. Finally, in the matching region, $\rho_{0} \ll(\omega R)^{2} \ll \rho \ll 1$, we have

$$
\phi(r) \rightarrow \alpha \rho^{l} / 2^{l+2}(l+2) !-\beta 2^{l+1}(l+1) ! / \pi \rho^{l+4} .
$$

Anticipating that for $\omega R \ll 1 \beta \rightarrow 0$, the absorption probability can be written as

$$
P(l)=2 \pi \lambda \rho_{0}^{4} /|\alpha|^{2}
$$

To study solutions of (3.6), it will be convenient to eliminate the first derivative term by introducing $u(x)=x^{-1 / 2} \sqrt{x^{2}-1} \phi(x)$ and rewriting (3.6) in the form

$$
u^{\prime \prime}=\left(\lambda^{2} F(x)+G(x)\right) u,
$$

where

$$
F(x)=-\frac{1}{4 x(1-x)^{2}(1+x)^{2}}, \quad G(x)=-\frac{x^{4}+6 x^{2}-3}{4 x^{2}\left(1-x^{2}\right)^{2}}+\frac{l(l+4)}{4 x^{2}\left(1-x^{2}\right)} .
$$

In the next subsection, we shall find solutions to equation (3.9) in the form of asymptotic series in $1 / \lambda$.

\subsection{Low-temperature expansion I: Langer - Olver method}

We are interested in constructing a uniform approximation to the solution of (3.9) on the interval $[0,1]$, the endpoints of the interval being regular singular points of the differential equation. Our expansion will be built around $x=0$, where the function $F(x)$ has a simple polef. The idea behind the method of Langer and Olver is to introduce new variables in which the behavior of the solution close to the singularity would be fully described (for large $\lambda$ ) by a known function, and treat the rest as a perturbation. In our case the situation is more complex since the interval of interest contains two singular points, and we have to prove that the functional coefficients of the expansion converge everywhere on $[0,1]$ - in particular, that they converge at $x=1$. The convergence can be established by analyzing the singularities of the functions $F(x)$ and $G(x)$ in (3.9) [14]. We also confirm it by an explicit calculation.

\footnotetext{
${ }^{3}$ The expansion cannot be constructed starting from $x=1$, since in that case the error-control function diverges (see remarks on p.205 of [14]).
} 
By making a conformal transformation $\zeta=-p^{2}(x)$, where

$$
p(x)=\frac{1}{2} \arctan \sqrt{x}+\frac{1}{4} \log \frac{1+\sqrt{x}}{1-\sqrt{x}}
$$

and introducing a new function $W(x)=x^{-1 / 4} \sqrt{p(x) /\left(x^{2}-1\right)} u(x)=x^{-3 / 4} \sqrt{p(x)} \phi(x)$ we transform equation (3.6) into

$$
\frac{d^{2} W}{d \zeta^{2}}=\left\{\frac{\lambda^{2}}{4 \zeta}+\frac{\nu^{2}-1}{4 \zeta^{2}}+\frac{\psi(\zeta)}{\zeta}\right\} W
$$

where $\nu=l+2$, and

$$
\psi(\zeta(x))=\frac{4 \nu^{2}-1}{16 p^{2}(x)}+\frac{\left(x^{2}-1\right)\left(9 x^{2}+4 \nu^{2}-1\right)}{16 x} .
$$

The function $\psi(\zeta) / \zeta$ is analytic at $\zeta=0(x=0)$ and is treated as a perturbation. Then the formal series solution of (3.11) corresponding to the wave propagating from the infinity to the horizon is given by

$W(x)=\sqrt{\frac{\pi \lambda}{2}} \frac{i^{l+2} p(x)}{N_{1}(\lambda, l)}\left\{H_{l+2}^{(1)}(\lambda p(x)) \sum_{n=0}^{\infty} A_{n}(x) \lambda^{-2 n}-\frac{p(x)}{\lambda} H_{l+3}^{(1)}(\lambda p(x)) \sum_{n=0}^{\infty} B_{n}(x) \lambda^{-2 n}\right\}$,

where $H_{\nu}^{(1)}(z)$ are Hankel functions. The coefficients $A_{n}, B_{n}$ are independent of $\lambda$ and can be calculated successively using the following equations [12]

$$
\begin{gathered}
A_{n+1}(\zeta)=\nu B_{n}(\zeta)-\zeta B_{n}^{\prime}(\zeta)+\int \psi(\zeta) B_{n}(\zeta) d \zeta \\
B_{n}(\zeta)=-A_{n}^{\prime}(\zeta)+\frac{1}{\sqrt{\zeta}} \int_{0}^{\zeta}\left\{\psi(t) A_{n}(t)-(\nu+1 / 2) A_{n}^{\prime}(t)\right\} \frac{d t}{\sqrt{t}}
\end{gathered}
$$

with $A_{0}=1$. The formal series in (3.13) provides a genuine asymptotic expansion under the condition that the successive integrals in (3.14), (3.15) are convergent \&. As can be readily seen ?, this is indeed the case for our $\psi(x)$, and therefore Olver's theorem guarantees that 3.13 ) is an asymptotic expansion approximating the solution of (3.11) uniformly for $x \in[0,1]$. In practice, it is far more convenient to use the following expressions for the coefficients $A_{n}(x), B_{n}(x)$ (see Chapter 12, Exercise 5.2 in [14]):

$$
A_{n}(x)=\sum_{k=0}^{2 n} \frac{i^{k} a_{k}(\nu+1)}{p^{k}(x)} \bar{A}_{2 n-k}(x)
$$

${ }^{4}$ Precise error bounds on partial sums of (3.13) can be found in Chapter 12, $\S 4$ of [14]). Conditions on $\psi$ sufficient (but not necessary!) for the convergence of the error-control function are stated in Exercise 4.2, Chapter 12 of 14].

${ }^{5}$ For $x \rightarrow 1$ we have $B_{n} \sim 1 / \log \epsilon, A_{n} \sim O(1)$, where $\epsilon=|x-1|$. 


$$
B_{n}(x)=i \sum_{k=0}^{2 n+1} \frac{i^{k} a_{k}(\nu)}{p^{k+1}(x)} \bar{A}_{2 n+1-k}(x)
$$

where

$$
\bar{A}_{k+1}(x)=i \sqrt{x}\left(x^{2}-1\right) \partial_{x} \bar{A}_{k}+i \int \frac{9 x^{2}+4 \nu^{2}-1}{16 x \sqrt{x}} \bar{A}_{k} d x,
$$

and $a_{k}(\nu)=\left(4 \nu^{2}-1^{2}\right)\left(4 \nu^{2}-3^{2}\right) \ldots\left(4 \nu^{2}-(2 k-1)^{2}\right) / 8^{k} k !$. The normalization constant $N_{1}(\lambda, l)$ in $(3.13)$,

$$
N_{1}(\lambda, l)=e^{i \lambda \log 2 / 2-i \pi / 4+i \lambda \pi / 8} \sum_{n=0}^{\infty}\left(A_{n}(1)-\frac{i}{\lambda} \lim _{x \rightarrow 1} p(x) B_{n}(x) \lambda^{-2 n}\right),
$$

is chosen in such a way that for $x \rightarrow 1$ we have $\phi(x)=x^{3 / 4} W / \sqrt{p(x)} \rightarrow(1-x)^{-i \lambda / 4}$. Then in the limit $x \rightarrow 0$ we obtain

$$
\phi(x) \rightarrow \sqrt{\frac{2 \lambda}{\pi}} \frac{i^{l}(l+1) ! 2^{l+1} N_{0}}{\lambda^{l+2} N_{1}} x^{-l / 2},
$$

where

$$
N_{0}(\lambda, l)=\sum_{n=0}^{\infty} \lambda^{-2 n}\left(A_{n}(0)-2 \nu B_{n}(0) \lambda^{-2}\right)
$$

which can be compared with $\alpha J_{l+2}(\rho) / \rho^{2}$ in the region $\rho \ll 1$ to get the coefficient $\alpha$. The absorption probability is calculated in a standard way, and we find

$$
P(l)=P_{0}(l) \frac{\left|N_{1}(\lambda, l)\right|^{2}}{\left|N_{0}(\lambda, l)\right|^{2}}
$$

where $P_{0}(l)$ is given by (2.1). Calculating the first few coefficients, we get $B_{0}(0)=0$, $\lim _{x \rightarrow 1} p(x) B_{0}(x)=\left(\nu^{2}-1\right) / 2, A_{1}(0)=\left(\nu^{2}-1\right)^{2} / 8$ (see also Appendix A $)$. Then the ratio $f_{l}(\lambda)=P(l) / P_{0}(l)$ is given by (2.3). Higher order terms can be calculated as a result of a lengthy but essentially straightforward procedure using the recurrence relations 3.16 3.18). The result (2.3) therefore potentially accounts for all power-like terms in the $\lambda \gg 1$ expansion. From our treatment of the $D 1-D 5$-brane system in Section 3 it will become clear that the Langer - Olver expansion approximates the exact solution reasonably well even for $\lambda \sim 1$. In fact, the first few terms of the asymptotic expansion (3.13) coincide with the Frobenius series solution of (3.11) at $x=0$.

The following assertions regarding the asymptotic expansion (2.3) appear to be true:

(1) It is an expansion in powers of $1 / \lambda^{4}$. In particular, the $1 / \lambda^{2}$ correction vanishes, in disagreement

\footnotetext{
${ }^{6}$ In [8], the potential was approximated by its Taylor expansion near one of the singular points. Such an approximation is non-uniform and cannot give an accurate matching. The approximation used in [9], 10] is essentially of the form $f(x)=x^{\alpha_{0}}(1-x)^{\alpha_{1}}$, where $\alpha_{0,1}$ are indices of the singularities at $x=0,1$, respectively. This approximation provides the correct result for $P$ at $\lambda=\infty$ (absorption probability at zero temperature) but it cannot be used to calculate finite-temperature corrections. We remark that the results of [9], [10] disagree with those obtained in [8].
} 
(2) For the $s$-wave $(\nu=2)$ power-like corrections in (2.3) vanish.

Being an asymptotic expansion in powers of $1 / \lambda$, formula (2.3) cannot account for the corrections of the form $\exp (-\lambda)$. In the next section we show that such terms do indeed exist. Unfortunately, an efficient recursive scheme for their calculation is not known [19. Our attempt to construct it seems to face considerable technical difficulties.

\subsection{Low-temperature expansion II: Reduction to a hypergeo- metric equation}

Since we are interested in studying solutions in the interval $[0,1]$, it seems plausible that singular points $x=0, x=1$ play the major role for such solutions, whereas the singular point $x=-1$ can be treated perturbatively. Indeed, neglecting the term with $G(x)$ for a moment, we can write (3.9) as

$$
u^{\prime \prime}=-\frac{\lambda^{2} u}{4 x(1-x)^{2} \eta(x)},
$$

where $\eta(x)=(1+x)^{2}$ varies smoothly from 1 to 4 for $x \in[0,1]$. If we treat $\eta$ as a constant, equation (3.23) can be solved exactly in terms of a hypergeometric function, and therefore one may hope that the solution of the original equation (3.9) can be successfully approximated by a series based on hypergeometric function. We shall now try to make these ideas precise.

First, we introduce a new variable, $\zeta(x) \in[0,1]$, and make a conformal transformation,

$$
\begin{aligned}
\zeta & =\tanh ^{2} p(x), \\
p(x) & =(\arctan \sqrt{x}+\operatorname{arctanh} \sqrt{x}) / 2, \\
U & =u \sqrt{d \zeta / d x},
\end{aligned}
$$

to write equation $(3.9)$ in the form

$$
U^{\prime \prime}=-\frac{\lambda^{2}}{4 \zeta(1-\zeta)^{2}} U+\mathcal{P} U
$$

where derivatives are now taken with respect to $\zeta$ and

$$
\mathcal{P}=\dot{x}^{2} g(x(\zeta))-\frac{1}{2}\{x, \zeta\} .
$$

Here $\dot{x}=d x / d \zeta=\sqrt{x}\left(1-x^{2}\right) \cosh ^{2} p(x) / \tanh p(x)$, and

$$
\{x, \zeta\}=\frac{\dddot{x}}{\dot{x}}-\frac{3}{2}\left(\frac{\ddot{x}}{\dot{x}}\right)^{2}
$$


denotes the Schwarz derivative. We have

$$
p(x)= \begin{cases}\sqrt{x}\left(1+x^{2} / 5+O\left(x^{4}\right)\right) & \text { for } x \rightarrow 0, \\ -\frac{1}{4} \log (1-x)+\log 2 / 2+\pi / 8+O(x-1) & \text { for } x \rightarrow 1\end{cases}
$$

and so

$$
\zeta(x)= \begin{cases}x-2 x^{2} / 3+O\left(x^{3}\right) & \text { for } x \rightarrow 0 \\ 1-2 \sqrt{1-x} e^{-\pi / 4} & \text { for } x \rightarrow 1 .\end{cases}
$$

Accordingly, the potential $\mathcal{P}(\zeta)$ becomes

$$
\mathcal{P}(\zeta)= \begin{cases}\left(\nu^{2}-1\right) / 4 \zeta^{2}+O(1) & \text { for } x \rightarrow 0 \\ -1 / 4(1-\zeta)^{2}+O(1) & \text { for } x \rightarrow 1\end{cases}
$$

Introducing the "regularized" potential, $\mathcal{P}_{R}=\mathcal{P}-\left(\nu^{2}-1\right) / 4 \zeta^{2}+1 / 4(1-\zeta)^{2}$, we can write equation (3.27) as

$$
U^{\prime \prime}=\left(-\frac{\lambda^{2}}{4 \zeta(1-\zeta)^{2}}+\frac{\nu^{2}-1}{4 \zeta^{2}}-\frac{1}{4(1-\zeta)^{2}}+\mathcal{P}_{R}\right) U
$$

where $\mathcal{P}_{R}$ is a regular function for $\zeta \in[0,1]$. We shall look for solutions of the equation (3.32) in the form $U=U_{0}(1+h(\zeta))$, where $U_{0}$ obeys

$$
4 \zeta^{2}(1-\zeta)^{2} U_{0}^{\prime \prime}+\left(\lambda^{2} \zeta+\zeta^{2}-\left(\nu^{2}-1\right)(1-\zeta)^{2}\right) U_{0}=0
$$

and for the error-control function $h(\zeta)$ one can derive the following integral equation

$$
h(\zeta)=\int_{\zeta}^{1} \mathcal{P}_{R}(1+h(\zeta)) U_{o}^{2}(\xi) \int_{\xi}^{\zeta} \frac{d t}{U_{0}^{2}(t)} d \xi
$$

The solution of (3.33) is given by

$$
U_{0}(\zeta)=(1-\zeta)^{\frac{1-i \lambda}{2}}(-\zeta)^{\frac{1+\nu}{2}}{ }_{2} F_{1}\left(\frac{\nu-i \lambda}{2}+\eta_{+}(\nu), \frac{\nu-i \lambda}{2}+\eta_{-}(\nu) ; 1-i \lambda ; 1-\zeta\right)
$$

where $\eta_{ \pm}(\nu)=\left(1 \pm \sqrt{\nu^{2}-1}\right) / 2$. Returning to the $x$ variable, the solution normalized to $(1-x)^{-i \lambda / 4}$ for $x \rightarrow 1$ is

$$
\begin{aligned}
\phi_{0}(x)= & C_{\lambda} x^{3 / 4} \cosh ^{i \lambda} p(x) \tanh ^{\nu+1 / 2} p(x) \\
& { }_{2} F_{1}\left(-i \lambda / 2+\nu / 2+\eta_{+},-i \lambda / 2+\nu / 2+\eta_{-} ; 1-i \lambda ; \operatorname{sech}^{2} p(x)\right),
\end{aligned}
$$

where $C_{\lambda}=\exp (i \lambda \log 2 / 2-i \pi \lambda / 8)$. For small $x$ (recall that $\left.x=\rho_{0}^{2} / \rho^{2}\right)$ one has

$$
\phi_{0} \rightarrow \frac{\rho^{l}}{\rho_{0}^{l}} \frac{C_{\lambda} \Gamma(\nu) \Gamma(1-i \lambda)}{\Gamma\left(-i \lambda / 2+\nu / 2 \eta_{+}(\nu)\right) \Gamma\left(-i \lambda / 2+\nu / 2+\eta_{-}(\nu)\right)} .
$$


This matches (for $\omega R \ll 1$ ) the outer region solution, $\alpha J_{\nu}(\rho) / \rho^{2} \sim \alpha \rho^{l} / 2^{l+2} \Gamma(l+3$ ), with

$$
\alpha=\frac{2^{\nu} C_{\lambda} \Gamma(\nu+1) \Gamma(\nu) \Gamma(1-i \lambda)}{\Gamma\left(-i \lambda / 2+\nu / 2+\eta_{+}(\nu)\right) \Gamma\left(-i \lambda / 2+\nu / 2+\eta_{-}(\nu)\right)} .
$$

The absorption probability (3.8) can be written as

$$
P=\frac{2 \pi \lambda}{[\nu !(\nu-1) !]^{2}} \frac{\left|\Gamma\left(\nu / 2+\eta_{+}(\nu)+i \lambda / 2\right) \Gamma\left(\nu / 2+\eta_{-}(\nu)+i \lambda / 2\right)\right|^{2}}{|\Gamma(1+i \lambda)|^{2}}\left(\frac{\omega r_{0}}{2}\right)^{2 \nu}
$$

where we used $|\Gamma(x+i y)|=|\Gamma(x-i y)|$. The zero-temperature limit (corresponding to $\lambda \rightarrow \infty)$ gives (see Appendix B)

$$
P=P_{0}\left(1+4 \nu\left(\nu^{2}-1\right) / 3 \lambda^{2}+\cdots\right)
$$

where $P_{0}$ is given by (2.1). We must not forget, however, that there are corrections to the result (3.39) coming from the function $h(\zeta)$ in (3.34). Standard analysis of the Volterra equation (3.34) suggests that $h(\zeta)$ is indeed a small correction (properties of $\mathcal{P}_{R}$ and $U_{0}$ ensuring convergence of corresponding integrals at the endpoints) of the order of $1 / \lambda^{2}$. Therefore only the zeroth order term in the expansion (3.40) can be trusted and thus there is no contradiction with our main result (2.3). The significance of (3.39) is twofold: it shows that the absorption probability must contain contributions of the form $\exp (-\lambda)$, and that it has a functional form expected in a field theory (compare (4.4)). We can try to guess the exact expression for the absorption probability by choosing the ansatz of the form!

$$
P=\frac{2 \pi \lambda}{[\nu !(\nu-1) !]^{2}} \frac{\left|\Gamma\left(x_{1}+i \lambda / 2\right) \Gamma\left(x_{2}+i \lambda / 2\right)\right|^{2}}{|\Gamma(1+i \lambda)|^{2}}\left(\frac{\omega r_{0}}{2}\right)^{2 \nu}
$$

Comparison with the asymptotic expansion (2.3) to the order $1 / \lambda^{2}$ gives

$$
x_{1,2}=\left(\nu+1 \pm \sqrt{\left(1-\nu^{2}\right) / 3}\right) / 2 .
$$

However, the coefficient in front of $1 / \lambda^{4}$ term turns out to be $32 \nu\left(\nu^{2}-1\right)\left(4 \nu^{2}-1\right) / 45$ which does not match (2.3). This suggests that the naive thermal ansatz (3.41) is incorrect.

\subsection{High-temperature expansion}

Two independent solutions of (3.6) are

$$
\phi_{ \pm}(x)=(1-x)^{\sigma_{ \pm}} F(x),
$$

\footnotetext{
${ }^{7}$ For the $D 1-D 5$ system this method gives the exact thermal factor in (4.4) immediately.
} 
where $\sigma_{ \pm}= \pm i \lambda / 4$ are the roots of the indicial equation and $F(x)$ obeys

$$
\begin{aligned}
\frac{d^{2} F}{d x^{2}} & -\frac{x^{2}(1+2 \sigma)+2 \sigma x+1}{x\left(1-x^{2}\right)} \frac{d F}{d x}-\frac{l(l+4)}{4 x^{2}\left(1-x^{2}\right)} F \\
& +\frac{x(1+x) \sigma(\sigma-1)+\sigma\left(1+x^{2}\right)}{x(1-x)^{2}(1+x)} F+\frac{\lambda^{2}}{4 x\left(1-x^{2}\right)^{2}} F=0 .
\end{aligned}
$$

The solution corresponding to the incoming wave at the horizon is

$$
\phi(r)=A\left(1-r_{0}^{2} / r^{2}\right)^{-i \lambda / 4} F(r)
$$

where $A$ is an arbitrary constant and $F(r)$ is supposed to be regular at $r=r_{0}(x=1)$. For $\lambda \ll 1$ the approximation scheme can be constructed applying a standard perturbation theory to the equation (3.43). In the zeroth-order approximation (3.43) reads

$$
x^{2}\left(1-x^{2}\right) F^{\prime \prime}-x\left(1+x^{2}\right) F^{\prime}-l(l+4) / 4 F=0,
$$

and has solutions

$$
\begin{aligned}
& F_{1}(x)=x^{2+l / 2}{ }_{2} F_{1}\left(1+l / 4,1+l / 4 ; 2+l / 2 ; x^{2}\right), \\
& F_{2}(x)=x^{-l / 2}{ }_{2} F_{1}\left(-l / 4,-l / 4 ;-l / 2 ; x^{2}\right) .
\end{aligned}
$$

We have to distinguish between even and odd $l$.

\section{Odd $l$ :}

The solution regular at $x=1$ (i.e. the one without logarithmic singularities) is given by $F(x)=F_{1}(x)+C_{l} F_{2}(x)$, where

$$
C_{l}=-\frac{\Gamma\left(2+\frac{l}{2}\right) \Gamma^{2}\left(-\frac{l}{4}\right)}{\Gamma\left(-\frac{l}{2}\right) \Gamma^{2}\left(1+\frac{l}{4}\right)}
$$

Matching this with the outer region's solution gives $\alpha=2^{l+2}(l+2) ! C_{l} / \rho_{0}^{l}$, and the absorption probability

$$
P=P_{0}(l)\left(\frac{2}{\lambda}\right)^{2 l+3} \frac{(l+1) !^{2}}{\pi\left|C_{l}\right|^{2}}=P_{0}(l)\left(\frac{2 \pi T}{\omega}\right)^{2 l+3} \frac{(l+1) !^{2}}{\pi\left|C_{l}\right|^{2}},
$$

where $P_{0}(l)$ is given by (2.1). 


\section{Even $l$ :}

In this case the solution regular at $x=1$ is $F_{2}(x)$. For $l=4 n, n=0,1,2, \ldots$, it is given by

$$
F_{2}(x)=\frac{n !^{2}}{(2 n) !} P_{n}\left(\frac{2}{x^{2}}-1\right)
$$

where $P_{n}(z)$ are the Legendre polynomials, and we have $F_{2}(x) \rightarrow x^{-l / 2}$ for $x \rightarrow 0$. For $l=4 n+2, n=0,1,2, \ldots$ the solution can be written in terms of the Meijer function [20]:

$$
F_{2}(x)=G_{20}^{20}\left(x^{2} \mid \begin{array}{ccc}
\cdot & 1 & 1 \\
-n-1 / 2 & n+3 / 2 & \cdot
\end{array}\right) .
$$

We have $F_{2}(x) \rightarrow x^{-l / 2} / \Gamma^{2}(1+l / 4)$ for $x \rightarrow 0$. Accordingly, the absorption probability is

$$
P=P_{0}(l)\left(\frac{2}{\lambda}\right)^{2 l+3} \frac{(l+1) !^{2}}{\pi}=P_{0}(l)\left(\frac{2 \pi T}{\omega}\right)^{2 l+3} \frac{(l+1) !^{2}}{\pi},
$$

for $l=0,4,8, \ldots$ and

$$
P=P_{0}(l)\left(\frac{2}{\lambda}\right)^{2 l+3} \frac{(l+1) !^{2} \Gamma^{4}(1+l / 4)}{\pi}=P_{0}(l)\left(\frac{2 \pi T}{\omega}\right)^{2 l+3} \frac{(l+1) !^{2} \Gamma^{4}(1+l / 4)}{\pi},
$$

for $l=2,6,10 \ldots$ Thus for the $s$-wave the absorption cross section $\sigma_{0}=32 \pi^{2} P(0) / \omega^{5}$ can be written as $\sigma_{0}=A\left(r_{0}, R\right) f(\lambda)$, where $A\left(r_{0}, R\right)=\pi^{3} r_{0}^{3} R^{2}$ is the horizon area of the three-brane $\mathrm{f}$ in the near-extremal limit, and $f(\lambda)$ interpolates between 1 at $\lambda=0$ and $\pi \lambda^{3} / 8$ at $\lambda=\infty$.

Corrections to the lowest-order results (3.47), (3.48), (3.49) can be obtained by applying regular perturbation theory to the equation (3.43). For example, for the $s$-wave we have

$F(x)=1-\frac{i \lambda}{4} \log \left(\frac{1+x}{2}\right)+\frac{\lambda^{2}}{32}\left[\left(\log \left(\frac{1+x}{2}\right)+8\right) \log \left(\frac{1+x}{2}\right)-4 \operatorname{Li}_{2}\left(\frac{1-x}{2}\right)\right]+O\left(\lambda^{3}\right)$.

Correspondingly, the absorption cross section becomes

$$
\sigma_{0}=\pi^{6} R^{8} T^{3}\left[1+\frac{\left(\pi^{2}+12(2-\log 2) \log 2\right) \omega^{2}}{48 \pi^{2} T^{2}}+\cdots\right] .
$$

The frequency-dependent term in (3.51) can be interpreted as a correction to a hydrodynamic description of the strongly coupled Yang-Mills plasma [22].

\footnotetext{
${ }^{8}$ This is reminiscent of the universal result for the black holes [21.
} 


\section{Absorption by the $D 1 / D 5$-branes}

The bound state of $D 1$ and $D 5$ branes has been intensively studied as a string theory model for a five-dimensional black hole, and later in the context of AdS/CFT correspondence (discussion and references can be found in [1]). The non-extremal absorption probability contains a thermal factor which can be calculated exactly using a (super)gravity description in the "dilute gas" regime. Here we would like to calculate this temperature-dependent coefficient by using Langer-Olver's approximation. We shall see that power-like terms at any given order of the low-temperature expansion are indeed correctly reproduced, but the corrections exponentially suppressed at low temperatures are not captured. We start with the "near region" equation (3.7) of [6], where we shall put $T_{L}=T_{R}=T_{H}=T$ for simplicity and use notations $x=r_{0}^{2} / r^{2}, \lambda=\omega / 2 \pi T$ :

$$
\frac{d^{2} \phi}{d x^{2}}-\frac{1}{(1-x)} \frac{d \phi}{d x}-\frac{l(l+2)}{4 x^{2}(1-x)} \phi+\frac{\lambda^{2}}{4 x(1-x)^{2}} \phi=0,
$$

Introducing a new function $u$ by $u=\sqrt{x-1} \phi$ one can write (4.1) in the form

$$
u^{\prime \prime}=\left(\lambda^{2} F+G\right) u,
$$

where $F=-1 / 4 x(1-x)^{2}, G=l(l+2) / 4 x^{2}(1-x)-1 / 4(1-x)^{2}$. Equation (4.1) has an exact solution given (up to an arbitrary constant) by

$$
\phi=x^{-l / 2}(1-x)^{-i \lambda / 2}{ }_{2} F_{1}(-(l+i \lambda) / 2,-(l+i \lambda) / 2 ; 1-i \lambda ; 1-x)
$$

which leads to the absorption probability [6]

$$
P(l)=\frac{2 \pi \lambda}{l !^{2}(l+1) !^{2}}\left(\frac{\omega r_{0}}{2}\right)^{2 l+2}\left|\frac{\Gamma^{2}\left(\frac{l+2}{2}-\frac{i \lambda}{2}\right)}{\Gamma(1-i \lambda)}\right|^{2} .
$$

The plane wave absorption cross-section is then obtained by multiplying (4.4) by $4 \pi / \omega^{3}$. In the low temperature limit $(\lambda \rightarrow \infty)$ we have

$$
\begin{aligned}
P(l) / P_{0}(l) & =1+\frac{\nu\left(\nu^{2}-1\right)}{3 \lambda^{2}}+\frac{\nu\left(\nu^{2}-1\right)\left(5 \nu^{3}-9 \nu^{2}-5 \nu+21\right)}{90 \lambda^{4}} \\
& +\frac{\nu\left(\nu^{2}-1\right)(\nu-2)(\nu-3)\left(35 \nu^{4}-14 \nu^{3}-80 \nu^{2}+314 \nu+465\right)}{5670 \lambda^{6}} \\
& +O\left(1 / \lambda^{6}\right)+O\left(e^{-\lambda}\right),
\end{aligned}
$$

where $\nu=l+1$, the absorption probability at zero temperature is given by

$$
P_{0}(l)=\frac{4 \pi^{2}}{l !^{2}(l+1) !^{2}}\left(\frac{\lambda \omega r_{0}}{4}\right)^{2 l+2}=\frac{\pi^{2} \omega^{4} r_{1}^{2} r_{5}^{2}}{4} \frac{\left(\omega^{2} r_{1} r_{5}\right)^{2 l}}{2^{4 l} l !^{2}(l+1) !^{2}},
$$


and we have used $T=r_{0} / 2 \pi r_{1} r_{5}$. Notice that in addition to the power-like corrections coming from gamma-functions in the numerator of (4.4), expression (4.5) also contains corrections of the form exp $(-\lambda)$ produced by $|\Gamma(1-i \lambda)|$ in the denominator (see Appendix B). In fact, for $l=0$ there are no power-like corrections at any order:

$$
P(0)=\left(\frac{\pi \lambda \omega r_{0}}{2}\right)^{2} \operatorname{coth} \frac{\pi \lambda}{2}=P_{0}(0)\left(1+2 e^{-\pi \lambda}+\cdots\right)
$$

\subsection{Low-temperature asymptotic expansion}

Using Langer-Olver's method, we would like to construct an approximate solution of (4.1) in the form of asymptotic series in powers of $1 / \lambda^{2}$, and compare it to the large $\lambda$ expansion of the exact solution. We shall use the notations of Section 3 throughout, with the exception of $\lambda=\omega / 2 \pi T$ and $\nu=l+1$. Applying the conformal transformation

$$
\begin{gathered}
\zeta=-\operatorname{arctanh}^{2} \sqrt{x}, \\
W=\frac{\operatorname{arctanh}^{1 / 2} \sqrt{x}}{x^{1 / 4} \sqrt{x-1}} u,
\end{gathered}
$$

we can write (4.2) as

$$
\frac{d^{2} W}{d \zeta^{2}}=\left\{\frac{\lambda^{2}}{4 \zeta}+\frac{\nu^{2}-1}{4 \zeta^{2}}+\frac{\psi(\zeta)}{\zeta}\right\} W
$$

where the function

$$
\psi(x)=\frac{1-4 \nu^{2}}{16 \zeta(x)}+\frac{(1-x)\left(1-4 \nu^{2}-x\right)}{16 x}
$$

is analytic at $x=0$. The formal series solution of (4.10) representing the incoming wave is given by

$$
\begin{aligned}
W(x) & =\frac{i^{l+1}}{N_{1}(\lambda, l)} \sqrt{\frac{\pi \lambda}{2}} \operatorname{arctanh} \sqrt{x}\left\{H_{l+1}^{(1)}(\lambda \operatorname{arctanh} \sqrt{x}) \sum_{n=0}^{\infty} A_{n}(x) \lambda^{-2 n}\right. \\
& \left.-\frac{\lambda}{\operatorname{arctanh} \sqrt{x}} H_{l+2}^{(1)}(\lambda \operatorname{arctanh} \sqrt{x}) \sum_{n=0}^{\infty} B_{n}(x) \lambda^{-2 n}\right\}
\end{aligned}
$$

where the normalization constant $N_{1}(\lambda, l)$,

$$
N_{1}(\lambda, l)=e^{i \lambda \log 2-i \pi / 4} \sum_{n=0}^{\infty}\left(A_{n}(1)+\frac{i}{\lambda} \lim _{x \rightarrow 1} \operatorname{arctanh} \sqrt{x} B_{n}(x) \lambda^{-2 n}\right)
$$

is chosen in such a way that

$$
\bar{\phi}=x^{1 / 4} \operatorname{arctanh}^{-1 / 2} \sqrt{x} W \rightarrow(1-x)^{-i \lambda / 2}
$$


for $x \rightarrow 1$, and thus $\bar{\phi}$ can be identified with the exact solution (4.3). Since for $x \rightarrow 0$

$$
\bar{\phi} \rightarrow \frac{N_{0} 2^{l} i^{l} l !}{N_{1} \lambda^{l+1}} x^{-l / 2}
$$

where

$$
N_{0}(\lambda, l)=\sum_{n=0}^{\infty} \lambda^{-2 n}\left(A_{n}(0)-2 \nu B_{n}(0) \lambda^{-2}\right),
$$

one can match with the outer region's solution $\alpha J_{l+1}(\rho) / \rho$ in the overlapping region $\rho \ll 1$, and obtain the absorption probability

$$
P(l)=\frac{4 \pi^{2}}{l !^{2}(l+1) !^{2}}\left(\frac{\lambda \omega r_{0}}{4}\right)^{2 l+2}\left|\frac{N_{1}}{N_{0}}\right|^{2}=P_{0}(l)\left|\frac{N_{1}}{N_{0}}\right|^{2} .
$$

The recursion relations for the coefficients (3.16) - (3.17) are now

$$
\bar{A}_{k+1}(x)=i \sqrt{x}(x-1) \partial_{x} \bar{A}_{k}+i \int \frac{4 \nu^{2}-1+x}{16 x \sqrt{x}} \bar{A}_{k} d x
$$

One finds

$$
B_{0}(x)=\frac{1-4 \nu^{2}}{8 \operatorname{arctanh}^{2} \sqrt{x}}-\frac{\sqrt{x}}{8 \operatorname{arctanh} \sqrt{x}}\left(1+\frac{1-4 \nu^{2}}{x}\right),
$$

so that $B_{0}(0)=l(l+2) / 6$, and $\lim _{x \rightarrow 1} \operatorname{arctanh} \sqrt{x} B_{0}(x)=\nu^{2} / 2-1 / 4$. Limiting values of a few higher-order coefficients can be found in Appendix A. Substitution into (4.17) gives precisely (4.5) as expected. Due to the simplicity of the function $\psi(x)$, the calculation of coefficients $A_{n}, B_{n}$ is straightforward, and therefore our result can in principle be extended to all orders in $1 / \lambda^{2}$. However, the asymptotic expansion (4.12) cannot capture $\exp (-\lambda)$ correction terms which in the $s$-wave case would lead to an erroneous conclusion that $P(0)=P_{0}(0)$ for non-zero temperature.

In Figure (1) we plot the exact and the approximate solutions of (4.1). Figure (2) shows the ratio $P(l) / P_{0}(l)$ (exact and approximate results) as a function of $1 / \lambda$. It is clear that the Langer-Olver approximation which is supposed to work well for large $\lambda$ is in fact quite good even for $\lambda \sim 1$. We remark that the Pade-improvement of the low-temperature asymptotic series (4.5) does not seem to be particularly helpful in this case.

\subsection{High-temperature expansion}

The leading term in the high-temperature expansion $(\lambda \ll 1)$ of $(4.4)$ is

$$
P=P_{0}(l)\left(\frac{2}{\lambda}\right)^{2 l+1} \frac{\Gamma^{4}(1+l / 2)}{\pi}=P_{0}(l)\left(\frac{4 \pi T_{H}}{\omega}\right)^{2 l+1} \frac{\Gamma^{4}(1+l / 2)}{\pi} .
$$


For the $s$-wave the absorption cross section is given by $\sigma_{0}=2 \pi^{2} r_{0} r_{1} r_{5} f(\lambda)$, where $f(\lambda)=$ $(\pi \lambda / 2) \operatorname{coth}(\pi \lambda / 2)$ interpolates between 1 at $\lambda=0$ and $\pi \lambda / 2$ at $\lambda=\infty$. Corrections can be readily obtained by solving equation (4.1) perturbatively in $\lambda \ll 1$. For the $s$-wave we get

$$
\phi(x)=(1-x)^{-i \lambda / 2}\left(1-\frac{\lambda^{2}}{4} \operatorname{Li}_{2}(1-x)+\cdots\right) .
$$

Correspondingly, we have for $l=0$ :

$$
P(0) / P_{0}(0)=2 / \pi \lambda+\pi \lambda / 6+O\left(\lambda^{2}\right) .
$$

For $l=1$ the result is

$$
P(1) / P_{0}(1)=\frac{\pi}{2 \lambda^{3}}+\frac{\pi}{\lambda}\left(1-\frac{\pi^{2}}{24}\right)+O(\lambda) .
$$

Of course, formulae (4.22), (4.23) coincide with the $\lambda \ll 1$ Taylor expansion of the exact result (4.4).

\section{$5 \quad$ Field theory calculation}

In field theory, the absorption cross section at finite temperature can be calculated using the method of thermal Green's functions (see e.g. [23]). The absorption cross section is determined by a discontinuity of the two-point function of the operator $\mathcal{O}_{l}$ to which the $l$ th partial wave of the dilaton field couples, and is given by the zero-momentum limit of the spectral density,

$$
\sigma_{l}^{T}=\lim _{k \rightarrow 0} \rho_{l}^{T}(\omega, k),
$$

where $\rho_{l}^{T}(\omega, k)$ at finite temperature in the imaginary time formalism is proportional to the discontinuity of the (analytically continued) Euclidean thermal two point function $\Pi(r, \tau)$,

$$
\rho_{l}^{T}(\omega, k)=\frac{\Omega}{\mathcal{F}} \int d^{3} x d t e^{-i \mathbf{k x}+i \omega t}[\bar{\Pi}(r, t-i \epsilon)-\bar{\Pi}(r, t+i \epsilon)] .
$$

Here the function $\bar{\Pi}(r, t)$ denotes the analytic continuation of $\Pi(r, \tau)$ to the real time $t=-i \tau, \mathcal{F}$ denotes the flux, and $\Omega$ is a coefficient which depends on the parameters of the system under consideration.

\section{$5.1 \quad$ D1/D5 system}

The absorption cross section is given by (5.1), (5.2), where $\bar{\Pi}(r, t)$ is the (analytically continued) two-dimensional thermal Green's function,

$$
\bar{\Pi}(r, t)=\frac{\mathcal{C}_{\mathcal{O}}}{i^{2 h_{L}+2 h_{R}}}\left(\frac{\pi T_{L}}{\sinh \pi T_{L}(t+x)}\right)^{2 h_{L}}\left(\frac{\pi T_{R}}{\sinh \pi T_{R}(t-x)}\right)^{2 h_{R}} .
$$




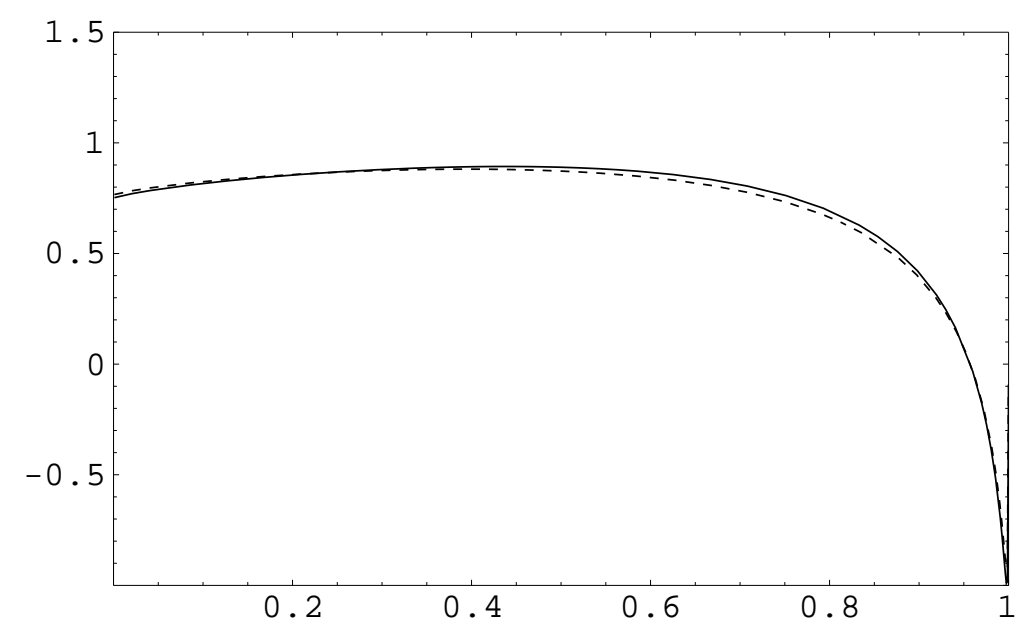

Figure 1: $D 1 / D 5$ system: exact solution $\operatorname{Re} \phi(x)$ (solid line) and a partial sum (with all $A_{s}, B_{s}$ equal to zero for $s>0$ ) of the asymptotic series for $\lambda=1$ (dashed line).

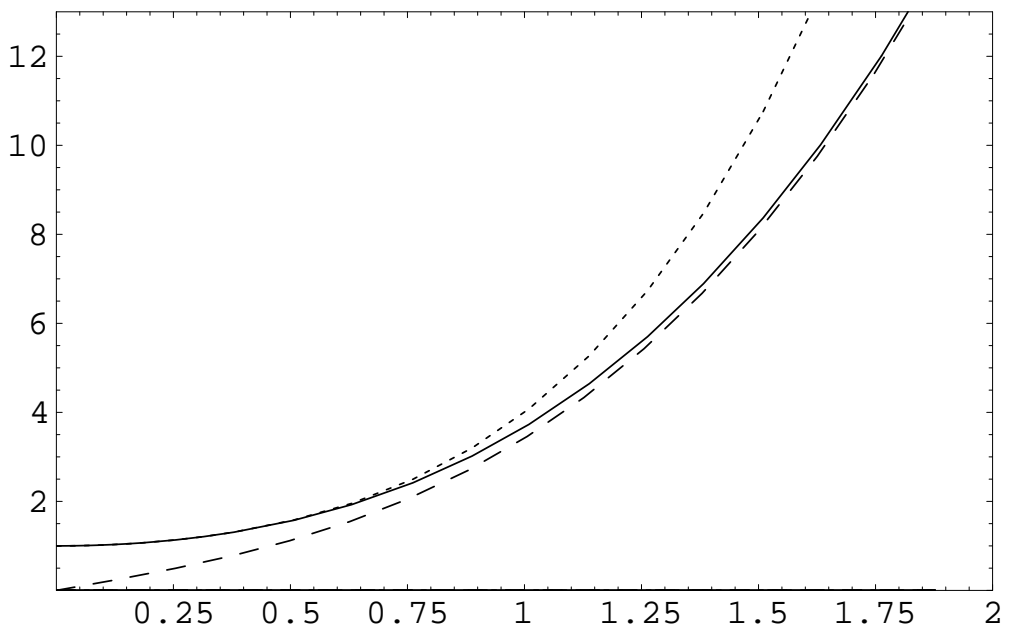

Figure 2: $D 1 / D 5$ system: ratio $P(l) / P_{0}(l)$ versus $1 / \lambda$ for $l=1$ : exact (solid line), high-temperature approximation (4.23) (dashed line), sum of the first three terms of the low-temperature asymptotic expansion (4.5) (dotted line). 
The integral in (5.2) factorizes nicely by introducing $x_{ \pm}=t \pm x$, and can be computed exactly 24]. This factorization is a property of a two-dimensional theory and does not hold in higher dimension. To illustrate the approach we shall use in the $d=4$ case we compute the $D 1 / D 5$ absorption cross section $\sigma_{0}^{T}$ by integrating over $t$ and $x$ directly without factorization. One can also show that the low-temperature asymptotic series (4.5) can be obtained by expanding $\bar{\Pi}(r, t)$ in powers of $T$. The spectral density for the $s$-wave is given by (5.2) with $\Omega=2 \pi r_{1}^{2} r_{5}^{2}, \mathcal{F}=\omega$ and

$$
\bar{\Pi}(r, t)=\frac{\pi^{4} T_{L}^{2} T_{R}^{2}}{\sinh ^{2} \pi T_{L}(t+x) \sinh ^{2} \pi T_{R}(t-x)} .
$$

Integrating over time we get

$$
\begin{aligned}
\rho_{0}^{T}(\omega, k) & =\frac{4 \pi^{4} r_{1}^{2} r_{5}^{2}}{\omega} \int d x e^{-i k x}\left[\frac{e^{-i \omega x} T_{R}^{2}\left(2 \pi i T_{R} \operatorname{coth}\left(2 \pi T_{R} x\right)-\omega\right)}{\sinh ^{2} 2 \pi T_{R} x}\right. \\
& \left.-\frac{e^{i \omega x} T_{L}^{2}\left(2 \pi i T_{L} \operatorname{coth}\left(2 \pi T_{L} x\right)+\omega\right)}{\sinh ^{2} 2 \pi T_{L} x}\right] .
\end{aligned}
$$

Evaluating residues, we obtain the spectral density

$$
\rho_{0}^{T}(\omega, k)=\frac{\pi^{2}\left(\omega^{2}-k^{2}\right)}{4}\left[\operatorname{coth} \frac{(\omega+k)}{4 T_{R}}+\operatorname{coth} \frac{(\omega-k)}{4 T_{L}}\right] .
$$

The absorption cross section is then obtained by taking the limit $k \rightarrow 0$ in (5.6),

$$
\sigma=\sigma_{0} f\left(\omega, T_{L}, T_{R}\right)=\pi^{3} r_{1}^{2} r_{5}^{2} \omega \frac{e^{\frac{\omega}{T_{H}}}-1}{\left(e^{\frac{\omega}{2 T_{L}}}-1\right)\left(e^{\frac{\omega}{2 T_{R}}}-1\right)}
$$

where $\sigma_{0}$ is the cross-section at zero temperature. Similar calculations can be done for higher partial waves. For all of them one observes a perfect agreement with the results obtained from gravity [4], [5], [6], [24].

\subsection{Non-extremal three-branes}

To the leading order in 't Hooft coupling, the terms contributing to $\left\langle\mathcal{O}_{l}(x) \mathcal{O}_{l}(0)\right\rangle$ are a bosonic term $\Pi_{l}^{\text {bos }}$ (for all $l \geq 0$ ), a two-fermion term $\Pi_{l}^{2 \Theta}$ (for $l \geq 1$ ), and a four-fermion term $\Pi_{l}^{4 \Theta}$ (for $l \geq 2$ ) identified in 25 :

$$
\Pi_{l, \mathrm{bos}}^{T=0}(x)=\frac{\kappa^{l} \omega^{2 l} N^{l+2}(l+2)(l+3)}{3 \pi^{l / 2} 2^{l+1} l !(l+1) !} \Delta^{l}(x)\left(\partial_{a} \partial_{b} \Delta(x) \partial_{a} \partial_{b} \Delta(x)+\frac{1}{2} \partial^{2} \Delta(x) \partial^{2} \Delta(x)\right)
$$

where $\Delta(x)=1 / 4 \pi^{2}|x|^{2}$ is the free scalar propagator. The term proportional to $\partial^{2} \Delta(x)$ does not contribute to the discontinuity and will be omitted. At finite temperature the 
bosonic contribution to the two-point function will be given by (5.8) with $\Delta(x)$ replaced by its thermal cousin. Borrowing the expression for the thermal propagator in position space from [26],

$$
\mathcal{G}_{B}(r, \tau)=\frac{T}{4 \pi r} \frac{\sinh 2 \pi r T}{(\cosh 2 \pi r T-\cos 2 \pi \tau T)},
$$

where $r=|\vec{x}|$, we obtain

$$
\Pi_{l, \mathrm{bos}}^{T}(r, \tau)=\frac{\kappa^{l} \omega^{2 l} N^{l+2}(l+2)(l+3)}{3 \pi^{l / 2} 2^{l+1} l !(l+1) !} \mathcal{M}_{l}(r, \tau),
$$

where

$$
\mathcal{M}_{l}(r, \tau)=\mathcal{G}_{B}^{l}\left[\left(\partial_{\tau \tau}^{2} \mathcal{G}_{B}\right)^{2}+\left(\partial_{r r}^{2} \mathcal{G}_{B}\right)^{2}+2\left(\partial_{r \tau}^{2} \mathcal{G}_{B}\right)^{2}+2\left(\partial_{r} \mathcal{G}_{B}\right)^{2} / r^{2}\right]
$$

For the $s$-wave we obtain (see Appendix 9 for details)

$$
\rho_{0}^{\mathrm{bos}}(\omega, k)=\frac{\pi k\left(\omega^{2}-k^{2}\right)^{2}}{32}\left[1+\frac{2 T}{k} \log \left(\frac{1-e^{-\frac{\omega+k}{2 T}}}{1-e^{-\frac{\omega+k}{2 T}}}\right)\right] .
$$

Taking the limit $k \rightarrow 0$ we get

$$
\sigma_{0}^{T}=\frac{\kappa^{2} N^{2} \omega^{3}}{32 \pi} \operatorname{coth} \frac{\omega}{4 T}=\sigma_{0}^{T=0} \operatorname{coth} \frac{\omega}{4 T},
$$

where $\kappa^{2} N^{2}=4 \pi^{5} R^{8}$.

For partial waves with $l>0$ in addition to (5.2) one has to take into account contributions of the two- and four-fermion operators. The contribution of the two-fermion operator is

$$
\Pi_{l, 2 \Theta}^{T}(r, \tau)=\frac{\kappa^{l} \omega^{2 l} N^{l+2}(l+2)(l+3)}{3 \pi^{l / 2} 2^{l}(l-1) !(l+1) !} \mathcal{M}_{l, 2 \Theta}(r, \tau),
$$

where

$$
\mathcal{M}_{l, 2 \Theta}(r, \tau)=\mathcal{G}_{B}^{l-1}\left[\partial_{r r}^{2} \mathcal{G}_{B}\left(\partial_{r} \mathcal{G}_{F}\right)^{2}+\partial_{\tau \tau}^{2} \mathcal{G}_{B}\left(\partial_{\tau} \mathcal{G}_{F}\right)^{2}+2 \partial_{r \tau}^{2} \mathcal{G}_{F} \partial_{r} \mathcal{G}_{F} \partial_{\tau} \mathcal{G}_{F}\right]
$$

and the free fermionic propagator at finite temperature is given by

$$
\mathcal{G}_{F}(r, \tau)=\frac{T}{2 \pi r} \frac{\sinh \pi r T \cos \pi T \tau}{(\cosh 2 \pi r T-\cos 2 \pi \tau T)} .
$$

Here we only consider the $l=1$ case. For general $l$ the calculation is similar but more tedious since the four-fermion operators also contribute. Expanding $\mathcal{M}_{1,2 \Theta}(r, \tau)$ in powers of $T$ and evaluating the discontinuity we get

$$
\sigma_{1, \text { field th }}^{T}=\frac{\kappa^{3} N^{3} \omega^{7}}{6144 \pi^{7 / 2}}\left[1+8\left(\frac{\pi T}{\omega}\right)^{2}+48\left(\frac{\pi T}{\omega}\right)^{4}+32\left(\frac{\pi T}{\omega}\right)^{6}+O\left(e^{-\omega / T}\right)\right] .
$$


Comparing this result with the one obtained from gravity,

$$
\sigma_{1, \mathrm{grav}}^{T}=\frac{\kappa^{3} N^{3} \omega^{7}}{6144 \pi^{7 / 2}}\left[1+48\left(\frac{\pi T}{\omega}\right)^{4}+O\left(e^{-\omega / T}\right)\right],
$$

we observe that $\sigma_{1, \text { field th }}^{T}$ contains terms proportional to $T^{2}$ and $T^{6}$ which are absent in $\sigma_{1, \text { grav }}^{T}$.

\section{$6 \quad$ Black $p$-branes and non-extremal $M$-branes}

In this Section we demonstrate that the $M$ - and black $p$-brane backgrounds can be treated by the approximation methods used in Section 2 provided the correct identification of the expansion parameter is made.

\subsection{Non-extremal $M$-branes}

Non-extremal $M$-brane solutions were found in [27]. In the notations of [28] the $M 2$-brane metric is given by

$$
d s_{11}^{2}=H^{-2 / 3}(r)\left(-f(r) d t^{2}+d x_{1}^{2}+d x_{2}^{2}\right)+H^{1 / 3}(r)\left(\frac{d r^{2}}{f(r)}+r^{2} d \Omega_{7}^{2}\right),
$$

where $H(r)=1+R^{6} / r^{6}, f(r)=1-r_{0}^{6} / r^{6}$. The radial part of the Laplace equation for a minimally coupled massless scalar propagating in the background (6.1) becomes

$$
\frac{d^{2} f}{d x^{2}}-\frac{\left(5 x^{3}+1\right)}{2 x\left(1-x^{3}\right)} \frac{d f}{d x}-\frac{l(l+6)}{4 x^{2}\left(1-x^{3}\right)} f+\frac{\lambda^{2}}{\left(1-x^{3}\right)^{2}}\left(1+\frac{\rho_{0}^{6}}{(\omega R)^{6} x^{3}}\right) f=0,
$$

where $x=r_{0}^{2} / r^{2}, \lambda^{2}=(\omega R)^{6} / 4 \rho_{0}^{4}, \rho=\omega r$. In the "inner" region $\rho \ll 1$ equation (6.2) is

$$
w^{\prime \prime}(x)=\left(-\frac{\lambda^{2}}{\left(1-x^{3}\right)^{2}}+\frac{l(l+6)}{4 x^{2}\left(1-x^{3}\right)}+\frac{5-46 x^{3}+5 x^{6}}{16 x^{2}\left(1-x^{3}\right)^{2}}\right) w(x)
$$

where $w(x)=f(x) x^{-1 / 4} \sqrt{x^{3}-1}$. Asymptotics of (6.3) can be constructed using LangerOlver's method. Here, however, the parameter of the expansion should be redefined according to $\lambda^{2} \rightarrow \Lambda^{2}=\lambda^{2}-3 l(l+6) / 4$ to ensure the convergence of the error-control functiong] [14].

\footnotetext{
${ }^{9}$ This redefinition is similar to the one performed in quantum mechanics where in quasi-classical approach to the central-force potentials a substitution $l(l+1) \rightarrow(l+1 / 2)^{2}$ is made "by hand" to achieve the correct asymptotic behavior of the solution.
} 
In the case of $M 5$-branes the metric is given by

$$
d s_{11}^{2}=\frac{-f(r) d t^{2}+d x_{1}^{2}+d x_{2}^{2}+d x_{3}^{2}+d x_{4}^{2}+d x_{5}^{2}}{H^{1 / 3}(r)}+H^{2 / 3}(r)\left(\frac{d r^{2}}{f(r)}+r^{2} d \Omega_{4}^{2}\right),
$$

where $H(r)=1+R^{3} / r^{3}, f(r)=1-r_{0}^{3} / r^{3}$. An analogue of the equation (6.2) is

$$
\frac{d^{2} f}{d x^{2}}-\frac{\left(x^{3}-2\right)}{x\left(1-x^{3}\right)} \frac{d f}{d x}-\frac{l(l+3)}{x^{2}\left(1-x^{3}\right)} f+\frac{\lambda^{2}}{x\left(1-x^{3}\right)^{2}}\left(1+\frac{\rho_{0}^{3}}{(\omega R)^{3} x^{3}}\right) f=0,
$$

where $x=r_{0} / r, \lambda^{2}=(\omega R)^{3} / \rho_{0}$. In the "inner" region, i.e. for $\rho \ll 1$ equation (6.5) becomes

$$
w^{\prime \prime}(x)=\left(-\frac{\lambda^{2}}{x\left(1-x^{3}\right)^{2}}+\frac{l(l+3)}{x^{2}\left(1-x^{3}\right)}+\frac{x\left(8-x^{3}\right)}{4\left(1-x^{3}\right)^{2}}\right) w(x)
$$

where $w(x)=f(x) x /\left(x^{3}-1\right)^{1 / 6}$. As before, an asymptotic expansion with the convergent coefficients can be built, provided the redefinition $\lambda^{2} \rightarrow \Lambda^{2}=\lambda^{2}-4-3 l(l+3)$ is made.

\subsection{Black $p$-branes:}

The metric is given by

$$
d s_{10}^{2}=\frac{-f(r) d t^{2}+d \vec{x} \cdot d \vec{x}}{\sqrt{H_{p}(r)}}+\sqrt{H_{p}(r)}\left(\frac{d r^{2}}{f(r)}+r^{2} d \Omega_{8-p}^{2}\right)
$$

where $H_{p}(r)=1+R^{7-p} / r^{7-p}, f(r)=1-r_{0}^{7-p} / r^{7-p}$. Let $\rho=\omega r$. Then the radial part of the Laplace equation for a minimally coupled massless scalar can be written as

$$
\frac{d^{2} f}{d \rho^{2}}+\left[a+1+\frac{a \rho_{0}^{a}}{\left(\rho^{a}-\rho_{0}^{a}\right)}+\frac{a(\omega R)^{a}(4-a)}{2\left(\rho^{a}+(\omega R)^{a}\right)}\right] \frac{1}{\rho} \frac{d f}{d \rho}-\frac{\rho^{a-2} l(l+a)}{\rho^{a}-\rho_{0}^{a}} f+\frac{\rho^{a}\left(\rho^{a}+(\omega R)^{a}\right)}{\left(\rho^{a}-\rho_{0}^{a}\right)^{2}} f=0,
$$

where $a=7-p, \rho_{0}=\omega r_{0}$. Solutions in the "outer" region (characterized by $\rho \ll \rho_{0}$, $\left.\rho \gg(\omega R)^{a /(a-2)}\right)$ are given by

$$
f(r)=\alpha \rho^{-a / 2} J_{l+a / 2}(\rho)+\beta \rho^{-a / 2} J_{-l-a / 2}(\rho)
$$

for odd $a$ and by

$$
f(r)=\alpha \rho^{-a} J_{l+a / 2}(\rho)+\beta \rho^{-a} Y_{l+a / 2}(\rho)
$$

for even $a$. To study solutions in the "inner" region, we introduce $x=r_{0}^{2} / r^{2}$ for $p$ branes with odd $p$ and $x=r_{0} / r$ for $p$-branes with even $p$. Then for the odd $p$-branes the

\footnotetext{
${ }^{10}$ The difference in treatment of even and odd $p$ reflects our desire to avoid fractional powers of $x$ in the potential.
} 
equation (6.8) becomes

$$
\begin{aligned}
& \frac{d^{2} f}{d x^{2}}+\left[\frac{2-a}{2 x}-\frac{a x^{a / 2}}{2 x\left(1-x^{a / 2}\right)}-\frac{a(4-a) x^{a / 2}(\omega R)^{a} / \rho_{0}^{a}}{4 x\left(1+x^{a / 2}(\omega R)^{a} / \rho_{0}^{a}\right)}\right] \frac{d f}{d x} \\
+ & \frac{\lambda^{2}}{x^{3-a / 2}\left(1-x^{a / 2}\right)^{2}}\left(1+\frac{\rho_{0}^{a}}{(\omega R)^{a} x^{a / 2}}\right) f-\frac{l(l+a)}{4 x^{2}\left(1-x^{a / 2}\right)} f=0,
\end{aligned}
$$

where $a=7-p$ and $\lambda^{2}=(\omega R)^{a} / 4 \rho_{0}^{a-2}$. For even $p$-branes we get

$$
\begin{array}{r}
\frac{d^{2} f}{d x^{2}}+\left[\frac{1-a}{x}+\frac{a x^{a-1}}{\left(1-x^{a}\right)}-\frac{a(4-a)(\omega R)^{a} x^{a} / \rho_{0}^{a}}{2 x\left(1+(\omega R)^{a} x^{a} / \rho_{0}^{a}\right)}\right] \frac{d f}{d x} \\
+\frac{\lambda^{2}}{x^{4-a}\left(1-x^{a}\right)^{2}}\left(1+\rho_{0}^{a} /(\omega R)^{a} x^{a}\right) f-\frac{l(l+a)}{x^{2}\left(1-x^{a}\right)} f=0,
\end{array}
$$

where $a=7-p$ and $\lambda^{2}=(\omega R)^{a} / \rho_{0}^{a-2}$. In the "inner" region, $\rho \ll 1$, equations (6.11) and (6.12), correspondingly, become

$$
\begin{aligned}
& \frac{d^{2} f}{d x^{2}}+\frac{\left(a^{2}-6 a+4-(a-2)^{2} x^{a / 2}\right.}{4 x\left(1-x^{a / 2}\right)} \frac{d f}{d x}+\frac{\lambda^{2}}{x^{3-a / 2}\left(1-x^{a / 2}\right)^{2}} f-\frac{l(l+a)}{4 x^{2}\left(1-x^{a / 2}\right)} f=0 \\
& \frac{d^{2} f}{d x^{2}}+\frac{a^{2}-6 a+2-\left(a^{2}-8 a+2\right) x^{a}}{2 x\left(1-x^{a}\right)} \frac{d f}{d x}+\frac{\lambda^{2}}{x^{4-a}\left(1-x^{a}\right)^{2}} f-\frac{l(l+a)}{x^{2}\left(1-x^{a}\right)} f=0
\end{aligned}
$$

Equations (6.13) and (6.14) have regular singular points at $x=0,1, \infty$ and a number of others on the unit circle. These equations are in this sense similar to the black three-brane equation and therefore can be treated by the approximation methods used in Section 3 .

\section{Conclusions}

In this paper we develop a reliable approximation scheme to compute high- and lowtemperature corrections to the absorption cross section of a minimally coupled massless scalar in the background of near-extremal black branes. The absorption cross section for the non-extremal three-branes is explicitly computed using this scheme, and compared with the field-theoretic calculation. Our main results are summarized in Section 2 .

A number of conceptual and technical points remain to be clarified. To construct a well-defined scheme based on a hypergeometric approximation (Section 3.2) or any modification thereof would be an important development by itself. It would allow one to control exponentially small corrections and therefore quantitatively compare field theory and gravity results (2.4) - 2.5) for the $s$-wave at low temperature. For $l=1$, it would be interesting to determine the origin of the disagreement between (2.6) and (2.7) for $T \ll \omega$. In field theory, the spectral densities of correlators with $l>0$ can in principle 
be computed exactly. In gravity, corrections to the leading high-temperature results for higher partial waves (3.47), (3.48), (3.49) can also be determined.

It may also be interesting to repeat our calculations for non-minimally coupled fields.

The approach outlined in Section 6 could be useful for studying the world-volume theories on M-branes, as well as lower-dimensional QCD (see Section 6.2 of [1] and references therein).

Finally, the results obtained in this paper may prove useful in determining transport coefficients of the strongly coupled finite-temperature gauge theory [22].

\section{A Coefficients $A_{n}, B_{n}$}

Here we provide explicit expressions for the boundary values of the first few coefficients $A_{n}, B_{n}$ defined by (3.16), (3.17).

D1-D5 brane system:

$$
\begin{aligned}
A_{0} & =1 \\
A_{1}(0) & =\left(32 \nu^{3}-12 \nu^{2}-32 \nu+3\right) / 192, \\
A_{1}(1) & =-\left(16 \nu^{4}-8 \nu^{2}+2\right) / 128, \\
A_{2}(0) & =\left(10240 \nu^{6}-44544 \nu^{5}-6800 \nu^{4}+132480 \nu^{3}+17800 \nu^{2}-87936 \nu-4185\right) / 737280, \\
A_{2}(1) & =\left(128 \nu^{8}+2176 \nu^{6}-4496 \nu^{4}+3704 \nu^{2}-807\right) / 49152 .
\end{aligned}
$$

Note that $\mathcal{B}_{n}$ below is defined as $\lim _{x \rightarrow 1} p(x) B_{n}(x)$.

$$
\begin{aligned}
& B_{0}(0)=\left(\nu^{2}-1\right) / 6 \\
& \mathcal{B}_{0}(1)=\left(2 \nu^{2}-1\right) / 4 \\
& B_{1}(0)=-\left(116 \nu^{4}-345 \nu^{2}+229\right) / 1920 \\
& \mathcal{B}_{1}(1)=-\left(16 \nu^{6}+64 \nu^{4}-134 \nu^{2}+33\right) / 768 .
\end{aligned}
$$

\section{Black three-brane system:}

$$
\begin{aligned}
A_{0} & =1 \\
A_{1}(0) & =0 \\
A_{1}(1) & =-\left(\nu^{2}-1\right)^{2} / 8 \\
A_{2}(0) & =\left(795+32768 \nu-420 \nu^{2}-40960 \nu^{3}-11760 \nu^{4}+8192 \nu^{5}+2880 \nu^{6}\right) / 40960 \\
A_{2}(1) & =\left(6801-39404 \nu^{2}+9648 \nu^{4}-2880 \nu^{6}+320 \nu^{8}\right) / 12280 \\
A_{3}(0) & =0 \\
A_{3}(1) & =\left(146474829-1016514774 \nu^{2}+1282758581 \nu^{4}\right.
\end{aligned}
$$




$$
\begin{aligned}
&\left.-553717340 \nu^{6}+95818800 \nu^{8}-5888960 \nu^{10}-24640 \nu^{12}\right) / 1135411200 \\
& B_{0}(0)=0 \\
& \mathcal{B}_{0}(1)=\left(\nu^{2}-1\right) / 2 \\
& B_{1}(0)=\left(\nu^{4}-5 \nu^{2}+4\right) / 5 \\
& \mathcal{B}_{1}(1)=\left(1344-9152 \nu^{2}+3520 \nu^{4}-320 \nu^{6}\right) / 15360 \\
& B_{2}(0)=0 \\
& \mathcal{B}_{2}(1)=\left(76248624-464811632 \nu^{2}+280281408 \nu^{4}\right. \\
&\left.-60890368 \nu^{6}+3942400 \nu^{8}+78848 \nu^{10}\right) / 302776320
\end{aligned}
$$

Higher coefficients can be rather easily obtained using Mathematica ${ }^{\mathrm{TM}}$ but the expression is complicated and will not be presented here.

\section{B Asymptotic expansion of $|\Gamma(x+i y)|$ for $|y| \rightarrow \infty$}

Using the Stirling expansion one can obtain the following formula:

$$
\begin{aligned}
|\Gamma(x+i y)| & =\sqrt{2 \pi} e^{-\pi|y| / 2}|y|^{x-1 / 2}\left\{1+x(x-1)(2 x-1) / 12|y|^{2}\right. \\
& +x\left(20 x^{5}-132 x^{4}+245 x^{3}-150 x^{2}+5 x+12\right) / 1440|y|^{4} \\
& +x\left(280 x^{8}-4284 x^{7}+23046 x^{6}-50337 x^{5}+42735 x^{4}\right. \\
& \left.\left.-2331 x^{3}-10801 x^{2}+252 x+1440\right) / 362880|y|^{6}+O\left(1 /|y|^{8}\right)\right\}
\end{aligned}
$$

Notice that for $x=0,1 / 2,1$ the expression in the curly brackets is identically equal to 1 (no power-like corrections). This is not a surprise since we have $|\Gamma(i x)|^{2}=\pi / x \sinh \pi x$, $|\Gamma(1 / 2+i x)|^{2}=\pi / \cosh \pi x$ and $|\Gamma(1+i x)|^{2}=\pi x / \sinh \pi x$. Another useful fact is the product expansion of $\Gamma(x+i y)$ :

$$
\Gamma(x+i y)=\xi e^{i \eta},
$$

where

$$
\begin{gathered}
\xi=\Gamma(x) \prod_{n=0}^{\infty}\left\{1+\frac{y^{2}}{(x+n)^{2}}\right\}^{-1 / 2} \\
\eta=y\left\{-\gamma_{E}+\sum_{n=1}^{\infty}\left(\frac{1}{n}-\frac{1}{y} \tan ^{-1} \frac{y}{x+n-1}\right)\right\} .
\end{gathered}
$$




\section{Integrals required for a thermal field theory cal- culation of the absorption cross section}

Our approach to evaluating relevant thermal field theory integrals and their low- and hightemperature asymptotics can be illustrated by the following example. We are interested in computing the integral

$$
I(b, c)=\int_{0}^{\infty} \frac{\cos b x}{x \sinh c x} d x=-\log \left(2 \cosh \frac{\pi b}{2 c}\right)=-\frac{\pi b}{2 c}-\log \left(1+e^{-\pi b / c}\right),
$$

where the right-hand side is defined in the sense of analytic continuation. To calculate

(C.1) exactly one can either take the limit $\alpha \rightarrow 0$ of the expression (2.5.47.1 (p. 469) of [20])

$$
\int_{0}^{\infty} \frac{x^{\alpha-1} \cos b x}{x \sinh c x} d x=\frac{\Gamma(\alpha)}{(2 c)^{\alpha}}\left\{\zeta\left[\alpha, \frac{1}{2}\left(1+\frac{i b}{c}\right)\right]+\zeta\left[\alpha, \frac{1}{2}\left(1-\frac{i b}{c}\right)\right]\right\}
$$

or evaluate the sum of residues with an appropriate prescription for the pole at $x=0$.

To obtain a "low-temperature" asymptotic, $c \rightarrow 0$, we can expand $\sinh c x$ in series, and then integrate using residues. Taking $c \rightarrow 0$ pushes all poles of $\sinh c x$ except $x=0$ to infinity, and thus our integration picks only the "polynomial" part of the exact result, $-\pi b / 2 c$. Exponentially suppressed terms are not captured. This, however, is exactly what we need to compare the field-theoretic absorption cross-section with the one obtained from gravity in the form of a low-temperature expansion.

The other limit, $c \rightarrow \infty$, is more subtle. In this limit, the poles of sinh $c x$ merge on the imaginary axis and one cannot approximate $\sinh c x$ by the exponent. One way to proceed is to rescale $z=c x$, expand $\cos b z / c$ for large $c$, and then integrate. This produces (in the zeroth-order approximation) the right answer, $-\log 2$, assuming the regularization provided by the integral

$$
\int_{0}^{\infty} \frac{x^{\alpha-1}}{\sinh x} d x=\frac{2^{\alpha}-1}{2^{\alpha-1}} \Gamma(\alpha) \zeta(\alpha)
$$

The spectral density of the two-point function (5.10) for $l=0$ is given by

$$
\rho_{0}^{T}(\omega, k)=\frac{\kappa^{2}}{\pi^{2} \omega k}(P(2 \pi T, \omega-k)-P(2 \pi T, \omega+k)),
$$

where

$$
P(a, b)=A(a, b)+B(a, b)+I(a, b)+J(a, b)+S(a, b)+T(a, b),
$$




$$
\begin{aligned}
A(a, b) & =\frac{3 a^{2}}{8} A_{4,2}(a, b)+\frac{a^{4}}{4}\left(1+\frac{\omega^{2}}{2 a^{2}}\right) A_{2,2}(a, b)+\frac{3 a^{4}}{8} A_{2,4}(a, b), \\
B(a, b) & =\frac{3 a^{2} \omega}{8} B_{3,2}(a, b)+\frac{a^{4} \omega}{4} B_{1,2}(a, b)+\frac{3 a^{4} \omega}{8} B_{1,4}(a, b), \\
I(a, b) & =\frac{3 a}{8} I_{5,1}(a, b)-\frac{a \omega^{2}}{8} I_{3,1}(a, b)+\frac{3 a^{3}}{8} I_{3,3}(a, b) \\
& +\frac{a^{5}}{8}\left(1-\frac{\omega^{2}}{a^{2}}\right) I_{1,3}(a, b)+\frac{3 a^{5}}{8} I_{1,5}(a, b), \\
J(a, b) & =\frac{3 a \omega}{8} J_{4,1}(a, b)+\frac{3 a^{3} \omega}{8} J_{2,3}(a, b), \\
S(a, b) & =\frac{3 \omega}{8} S_{5}-\frac{\omega^{3}}{8} S_{3}, \\
T(a, b) & =-\frac{3 \omega^{2}}{8} T_{4},
\end{aligned}
$$

and the integrals involved are defined by

$$
\begin{array}{rlrl}
A_{m, n}(a, b) & =\int \frac{\cos b r d r}{r^{m} \sinh ^{n} a r}, \quad B_{m, n}(a, b)=\int \frac{\sin b r d r}{r^{m} \sinh ^{n} a r} \\
I_{m, n}(a, b) & =\int \frac{\cosh a r \cos b r d r}{r^{m} \sinh ^{n} a r}, & J_{m, n}(a, b)=\int \frac{\cosh a r \sin b r d r}{r^{m} \sinh ^{n} a r} \\
S_{m} & =\int \frac{\sin b r d r}{r^{m}}, & T_{m}=\int \frac{\cos b r d r}{r^{m}}
\end{array}
$$

Calculation gives

$$
\begin{aligned}
I_{5,1}(a, b) & =\frac{\pi b\left(8 a^{4}+20 a^{2} b^{2}-3 b^{4}\right)}{360 a}+\frac{2 a^{4} p_{5}}{\pi^{4}} \\
I_{3,1}(a, b) & =\frac{b\left(-2 a^{2}+b^{2}\right) \pi}{6 a}-\frac{2 a^{2} p_{3}}{\pi^{2}} \\
I_{3,3}(a, b) & =-\frac{\pi b\left(b^{4}-8 a^{4}\right)}{120 a^{3}}+\frac{12 a^{2} p_{5}}{\pi^{4}}+\frac{6 a b p_{4}}{\pi^{3}}+\frac{b^{2} p_{3}}{\pi^{2}} \\
I_{1,3}(a, b) & =\frac{\pi b^{3}}{6 a^{3}}-\frac{2 p_{3}}{\pi^{2}}-\frac{2 b p_{2}}{\pi a}-\frac{b^{2} p_{1}}{a^{2}}, \\
I_{1,5}(a, b) & =-\frac{\pi b^{3}\left(20 a^{2}+3 b^{2}\right)}{360 a^{5}}+\frac{2 p_{5}}{\pi^{4}}+\frac{2 b p_{4}}{\pi^{3} a}+\left(\frac{2}{3 \pi^{2}}+\frac{b^{2}}{\pi^{2} a^{2}}\right) p_{3} \\
& +\left(\frac{b^{3}}{3 \pi a^{3}}+\frac{2 b}{3 \pi a}\right) p_{2}+\left(\frac{b^{4}}{12 a^{4}}+\frac{b^{2}}{3 a^{2}}\right) p_{1},
\end{aligned}
$$




$$
\begin{aligned}
J_{2,3}(a, b) & =-\frac{\pi a}{15}+\frac{\pi b^{4}}{24 a^{3}}+\frac{6 a p_{4}}{\pi^{3}}+\frac{b p_{3}}{\pi^{2}}+\frac{b^{2} p_{2}}{\pi a} \\
J_{4,1}(a, b) & =-\frac{\pi a^{3}}{45}-\frac{\pi a b^{2}}{6}+\frac{\pi b^{4}}{24 a}+\frac{2 a^{3} p_{4}}{\pi^{3}} \\
A_{4,2}(a, b) & =-\frac{\pi b\left(24 a^{4}+20 a^{2} b^{2}+3 b^{4}\right)}{360 a^{2}}-\frac{8 a^{3} p_{5}}{\pi^{4}}-\frac{2 a^{2} b p_{4}}{\pi^{3}} \\
A_{2,2}(a, b) & =\frac{\pi b\left(2 a^{2}+b^{2}\right)}{6 a^{2}}+\frac{4 a p_{3}}{\pi^{2}}+\frac{2 b p_{2}}{\pi}, \\
A_{2,4}(a, b) & =-\frac{\pi b\left(88 a^{4}+40 a^{2} b^{2}+3 b^{4}\right)}{360 a^{4}}-\frac{8 a p_{5}}{\pi^{4}}-\frac{6 b p_{4}}{\pi^{3}}-\left(\frac{8 a}{3 \pi^{2}}+\frac{3 b^{2}}{\pi^{2} a}\right) p_{3} \\
& -\left(\frac{b^{3}}{3 a^{2} \pi}+\frac{4 b}{3 \pi}\right) p_{2}, \\
B_{1,2}(a, b) & =-\frac{\pi\left(2 a^{2}+3 b^{2}\right)}{6 a^{2}}+\frac{2 p_{2}}{\pi}+\frac{2 b p_{1}}{a} \\
B_{3,2}(a, b) & =\frac{\pi\left(8 a^{4}+20 a^{2} b^{2}+5 b^{4}\right)}{120 a^{2}}-\frac{6 a^{2} p_{4}}{\pi^{3}}-\frac{2 a b p_{3}}{\pi^{2}} \\
B_{1,4}(a, b) & =\frac{\pi\left(88 a^{4}+120 a^{2} b^{2}+15 b^{4}\right)}{360 a^{4}}-\frac{2 p_{4}}{\pi^{3}}-\frac{2 b p_{3}}{\pi^{2} a}-2\left(\frac{2}{3 \pi}+\frac{b^{2}}{2 \pi a^{2}}\right) p_{2} \\
& \left.-\frac{b^{3}}{6 a^{3}}\right) p_{1}, \\
& =\left(\frac{2 b}{3 a}\right)
\end{aligned}
$$

where $p_{n}(a, b)=\operatorname{Li}_{n}[\exp (-\pi b / a)]$ is a polylogarithm [20]. We also have $S_{3}=-\pi b^{2} / 2$, $S_{5}=\pi b^{4} / 24, T_{5}=\pi b^{3} / 6$.

\section{Acknowledgments}

We would like to thank P. A. Grassi, M. Porrati and D.T. Son for useful discussions and comments, and B. Bajc and F. W. J. Olver for correspondence. G.P. is supported in part by the Fondazione A. Della Riccia.

\section{References}

[1] O. Aharony, S. S. Gubser, J. Maldacena, H. Ooguri and Y. Oz, "Large N field theories, string theory and gravity," Phys. Rept. 323, 183 (2000) hep-th/9905111. 
[2] I. R. Klebanov, "World-volume approach to absorption by non-dilatonic branes," Nucl. Phys. B496, 231 (1997) hep-th/9702076.

[3] S. S. Gubser and I. R. Klebanov, "Absorption by branes and Schwinger terms in the world volume theory," Phys. Lett. B 413, 41 (1997) [hep-th/9708005.

[4] S. R. Das and S. D. Mathur, "Interactions involving D-branes," Nucl. Phys. B482, 153 (1996) hep-th/9607149.

[5] S. R. Das and S. D. Mathur, "Comparing decay rates for black holes and D-branes," Nucl. Phys. B478, 561 (1996) hep-th/9606185.

[6] J. Maldacena and A. Strominger, "Universal low-energy dynamics for rotating black holes," Phys. Rev. D 56, 4975 (1997) [hep-th/9702015.

[7] J. Maldacena, "D-branes and near extremal black holes at low energies," Phys. Rev. D 55, 7645 (1997) hep-th/9611125.

[8] Y. Satoh, "Propagation of scalars in non-extremal black hole and black p-brane geometries," Phys. Rev. D 58, 044004 (1998) hep-th/9801125.

[9] S. Musiri and G. Siopsis, Phys. Lett. B 504, 314 (2001) hep-th/0003284.

[10] J. F. Vazquez-Poritz, "Absorption by nonextremal D3-branes," hep-th/0007202.

[11] R. Langer, "On the asymptotic solutions of ordinary differential equations, with reference to the Stokes' phenomenon about a singular point", Trans. Amer. Math. Soc. 37 no. 3, 397-416 (1935).

[12] F. W. J. Olver, "The asymptotic solution of linear differential equations of the second order in a domain containing one transition point", Philos. Trans. Roy. Soc. London. Ser. A. 249, 65-97 (1956), "Uniform asymptotic expansions of solutions of linear second-order differential equations for large values of a parameter", Philos. Trans. Roy. Soc. London. Ser. A 250 479-517 (1958).

[13] C.A. Swanson, "Differential equations with singular points", Tech. Rep. 16. Department of Mathematics, California Institute of Technology, Pasadena, 1956. 24 pp., D.P. Kostomarov, "On the asymptotic behavior of solutions of certain linear differential equations of second order containing a large parameter" (Russian), Mat. Sb. N.S. 45(87) 17-30 (1958).

[14] F.W.J. Olver, Asymptotics and Special Functions, (A K Peters, Wellesley, 1997). 
[15] S. S. Gubser, "Can the effective string see higher partial waves?," Phys. Rev. D 56, 4984 (1997) hep-th/9704195.

[16] G. T. Horowitz and A. Strominger, "Black strings and P-branes," Nucl. Phys. B 360, 197 (1991).

[17] E. Kamke, Differentialgleichungen, Losungsmethoden und Losungen, (Akademische Verlagsgesellschaft Geest \& Portig, Leipzig, 1959).

[18] S. S. Gubser and A. Hashimoto, "Exact absorption probabilities for the D3-brane," Commun. Math. Phys. 203, 325 (1999) hep-th/9805140.

[19] F. W. J. Olver, private communication.

[20] A.P. Prudnikov, Yu.A. Brychkov and O.I. Marichev, Integraly i ryady. Specialnye funktsii (Nauka, Moscow, 1983). English translation: Integrals and Series. Vol.2: Special Functions; Vol.3: More Special Functions (Gordon \& Breach Science Publishers, New York, 1988-92).

[21] S. R. Das, G. Gibbons and S. D. Mathur, "Universality of low energy absorption cross sections for black holes," Phys. Rev. Lett. 78, 417 (1997) [hep-th/9609052.

[22] G. Policastro, D. T. Son and A. O. Starinets, "The shear viscosity of strongly coupled N = 4 supersymmetric Yang-Mills plasma," Phys. Rev. Lett. 87, 081601 (2001) hepth/0104066.

[23] A.A. Abrikosov, L.P. Gorkov and I.E. Dzyaloshinskii, Methods of quantum field theory in statistical mechanics (Prentice-Hall, Englewood Cliffs, New Jersey, 1963).

[24] S. S. Gubser, "Absorption of photons and fermions by black holes in four dimensions," Phys. Rev. D 56, 7854 (1997) hep-th/9706100.

[25] I. Klebanov, W. I. Taylor and M. Van Raamsdonk, "Absorption of dilaton partial waves by D3-branes," Nucl. Phys. B 560, 207 (1999) hep-th/9905174.

[26] A. S. Vshivtsev, V. Ch. Zhukovsky and A. O. Starinets, "Thermal Green's functions of massive scalar particles for finite matter density," Russ. Phys. J. 34, 589 (1991).

[27] R. Gueven, "Black p-brane solutions of D = 11 supergravity theory," Phys. Lett. B276, 49 (1992).

[28] M. Cvetic and A. A. Tseytlin, "Non-extreme black holes from non-extreme intersecting M-branes," Nucl. Phys. B478, 181 (1996) hep-th/9606033. 